\title{
La fundación de la Biblioteca Nacional del Perú en 1821
}

\author{
The Founding of the National Library of Peru in 1821
}

\author{
Pedro M. Guibovich Pérez \\ Pontificia Universidad Católica del Perú, Perú \\ pguibovich@pucp.edu.pe \\ (D) https://orcid.org/0000000306815908
}

\section{Resumen:}

La fundación de la Biblioteca Nacional del Perú fue uno de los hitos culturales más importantes en el proceso de establecimiento del nuevo estado peruano después de la proclamación de su independencia por el General José de San Martín en 1821. En este estudio propongo que la creación de la Biblioteca Nacional tiene una doble dimensión institucional y simbólica: constituye un hito en la constitución del nuevo estado y en el proceso de ruptura con el régimen colonial.

Palabras Clave: Biblioteca Nacional del Perú, José de San Martín, Independencia, Bibliotecas coloniales, Nación, Perú.

\begin{abstract}
:
The founding of the National Library of Peru was one of the most important cultural milestones in the process of establishing the new Peruvian state after the proclamation of its independence by General José de San Martín in 1821. In this study, I propose that the creation of the National Library has a double institutional and symbolic dimension: it constitutes a milestone in the constitution of the new state and in the process of breaking with the colonial regime.

KEYWORDS: National Library of Peru, Jose de San Martin, Independence, Colonial Libraries, Nation, Perú.
\end{abstract}

\section{INTRODUCCIÓN}

La guerra que puso fin al vínculo colonial que hacía del Virreinato del Perú parte del imperio español se desarrolló entre 1820 y 1824. El 8 de setiembre de 1820, una expedición marítima procedente de Chile llegó a la bahía de Paracas, 250 kilómetros al sur de Lima, con el propósito de derrotar a las tropas que sostenían el poder político español. Al mando de dicha expedición se encontraba el general José de San Martín. Tiempo después, se trasladó por mar al norte y desembarcó en Huacho, para desde allí poner sitio a la capital. Ante la proximidad del ejército patriota, el virrey José de la Serna abandonó Lima. En tal situación, a mediados de julio de 1821, San Martín ingresó en Lima y el 28 de ese mismo mes llevó a cabo la solemne proclamación de la Independencia. Cinco días después, el 3 de agosto suscribió un decreto en el cual unía en su persona el mando político y militar de todo el Perú libre, bajo el título de Protector. Se trataba, como es conocido, de un ordenamiento temporal ya que solo tendría vigencia hasta la reunión de una representación nacional que determinase la forma de gobierno. El 28 de agosto, San Martín suscribió el decreto que dispuso el establecimiento de una "Biblioteca Nacional" en Lima. Vista a la distancia y en una perspectiva histórica, dicha medida llama la atención porque entonces el régimen del Protectorado hacía poco que se había instaurado y militarmente era débil. Mientras tanto, el virrey de la Serna se hacía fuerte en la sierra central gracias a la movilización de recursos humanos y materiales y, sustentado en ellos, habría de mantener en jaque a las tropas patriotas por un largo tiempo.

Si hay un hecho repetido hasta la saciedad en textos y manuales de historia al tratar el régimen del Protectorado, es la fundación de la Biblioteca Nacional. De tanto repetirlo ha pasado a convertirse en uno de los actos más representativos de dicho régimen, con el consecuente olvido de muchas otras acciones no 
menos importantes de carácter económico, social, político y administrativo promulgadas por San Martín y sus ministros. No obstante las reiteradas alusiones a la fundación de la Biblioteca Nacional, es muy poco lo que sabemos de su historia inicial, debido a dos causas principales. En primer lugar, el escaso desarrollo de las investigaciones dedicadas a la historia del libro y las bibliotecas en el periodo tardío colonial e inicios del periodo independiente. ${ }^{1}$ En segundo lugar, la desaparición del archivo administrativo de la Biblioteca Nacional. ${ }^{2}$ Los estudios sobre los años iniciales de su funcionamiento se limitan básicamente a los realizados por Alberto Tauro (1951), Estuardo Núñez (1971) y Roger Saravia (2020). Hace más de medio siglo, Tauro del Pino, quien fue director de la Biblioteca Nacional, dio a conocer valiosos datos relacionados con las acciones administrativas y los donativos de libros que hicieron posible el establecimiento de la institución entre 1821 y 1822. Por su parte, Núñez reconstruyó la trayectoria biográfica de su primer bibliotecario, el clérigo Mariano José de Arce. En tiempos más recientes, Saravia ha sostenido que el primer director y promotor de la biblioteca fue Bernardo de Monteagudo, ministro de Estado durante el Protectorado. Dada la imposibilidad de avanzar en el proyecto de reconstruir la historia institucional debido a la inexistencia de fuentes primarias, se propone explorar una cuestión no menos importante: el significado de la fundación de la Biblioteca Nacional en el contexto histórico de la guerra por la Independencia.

A lo largo de los siglos, la biblioteca ha significado muchas cosas, escribió Alice Crawford (2015): una colección de libros, un centro para la investigación académica, una memoria universal, un laberinto, un repositorio de conocimiento oculto o escondido, un santuario, un archivo para las historias, una fortaleza, un espacio de trascendencia. La misma autora sostiene: "A concept that has inspired many methaphors, the library as an idea has appealed to the human imagination throughout the ages and continuos to do so today" (Crawford, 2015, p. xvii).

Los hombres de la Ilustración creían firmemente en el poder transformador de los libros. Para Nicolás de Condorcet y Thomas Jefferson, la difusión de los libros y el entendimiento de la palabra impresa eran poderosas fuerzas para la liberación de la humanidad (Darnton, 2015). No extraña, entonces, que los padres fundadores del Estado peruano, hijos de la Ilustración, asignaran a la cultura letrada un poder transformador y que, como sugiere Crawford, imaginaran que con el establecimiento de la Biblioteca Nacional debía iniciarse un régimen cultural diferente al de la colonia. En las páginas que siguen se afirma que la creación de la Biblioteca Nacional tiene una doble dimensión institucional y simbólica: constituye un hito en la constitución del nuevo Estado y en el proceso de ruptura con el régimen colonial.

\section{Bibliotecas coloniales}

Al nuevo Estado le correspondían nuevas instituciones, y la Biblioteca Nacional fue la primera de su tipo en territorio peruano. Antes de agosto de 1821, en Lima solo existían bibliotecas (o "librerías", como se les llamaba) de carácter privado. Desde el siglo XVI, las principales órdenes religiosas - agustina, mercedaria, franciscana, dominica y jesuita - habían constituido por compras y donativos enormes colecciones de libros para el estudio y la consulta de sus miembros. También una elite de médicos, juristas, artesanos, curacas y clérigos, con capacidad económica e interés por el cultivo del intelecto, compraban libros tanto nuevos como usados. En los estantes de las bibliotecas conventuales y privadas se alineaban textos producidos en las prensas de los mayores centros editoriales del Viejo Continente como Lyon, Frankfurt, París, Colonia, Roma, Madrid, entre otros. Y en mucha menor proporción estaban representados los impresos salidos de los talleres tipográficos americanos. En el mundo colonial, conviene recordar, el libro que se leía era preferentemente el procedente de Europa. Los libros circulaban de unas manos a otras, de unos estantes a otros, se leían de forma privada y pública, y se atesoraban con especial cuidado. ${ }^{3}$ Pero en el siglo XVIII, al cultor de la lectura se le presentaron mayores oportunidades con la creación de bibliotecas públicas. 
La creación de las bibliotecas públicas fue un fenómeno cultural que se dio en Europa, y en particular en Francia, durante el siglo XVIII. París, por ejemplo, contaba en 1784 con dieciocho colecciones abiertas al público (Chartier, 1993). En América del sur, la apertura de bibliotecas públicas se dio en el contexto de las llamadas reformas borbónicas. Los funcionarios de la corona española promovieron el comercio, la manufactura, la explotación de los recursos naturales, pero también fomentaron el conocimiento mediante el establecimiento de las Sociedades económicas y la producción editorial en España (López Vidriero, 1994; Shafer, 1958). En ese contexto, primero en Bogotá en 1777 y luego en Quito en 1792, se crearon bibliotecas públicas a partir de los fondos bibliográficos que habían pertenecido a la Compañía de Jesús.

A mediados del siglo XVIII, dada su condición de centro político, económico y cultural, Lima era el principal mercado de consumo libresco y sus bibliotecas, las más ricas en el extenso virreinato peruano. Precisamente en esas magníficas colecciones limeñas pensó José Eusebio Llano Zapata (2005), cuando propuso la organización de una "biblioteca pública" a cargo de la Universidad de San Marcos, la cual debía funcionar en su local. A pesar de su condición de ilegitimidad, ya que era hijo de un sacerdote, cursó estudios en el afamado colegio de San Buenaventura, regentado por los franciscanos en Lima. En esta misma ciudad, frecuentó a los más importantes hombres de letras y de ellos aprendió el cultivo de las humanidades y la pasión por los libros. Movido por un afán de lograr algún puesto en la administración imperial, en 1750 dejó Lima con destino a España. En su viaje pasó por Chile, y en 1751 se detuvo en Mendoza y después en Buenos Aires, donde por cinco años fue huésped del obispo de esa ciudad, Cayetano Marcellano de Agramont. Se sirvió de la biblioteca del prelado para profundizar sus estudios sobre la historia de la América meridional. En 1755 partió para Cádiz. En su residencia gaditana formó una extensa biblioteca, resultado de su insaciable curiosidad, y practicó intensamente el género epistolar con otros cultores de la ciencia, la literatura y la historia como un medio para alcanzar conocimiento, pero sobre todo un lugar en la República de las Letras. En vida, publicó numerosas cartas y dejó inéditos varios trabajos sobre los recursos naturales y la historia peruana. Falleció en 1780 (Walker, 2005).

En una carta a Marcellano de Agramont, por entonces arzobispo de La Plata, suscrita en Cádiz el 30 de junio de 1758, Llano Zapata (2005, pp. 594-595) se quejaba de las dificultades que enfrentaban los que escribían sobre América por carecer Lima de "una biblioteca pública que estuviese abierta a sus horas para el libre uso". El principal objetivo de la institución debía ser conservar "todos los originales de nuestros sucesos que se pudiesen haber a las manos”. Para la conformación del fondo bibliográfico, Llano Zapata planeó que los candidatos a algún grado académico o una plaza de catedrático en la Universidad de San Marcos debían entregar libros para la constitución de la biblioteca pública. El propio escritor limeño ofreció donar 500 volúmenes. Asimismo, debía solicitarse donativos a los grandes propietarios de libros en Lima. Pero, como bien lo ha demostrado Jorge Solís (1993), el proyecto de Llano Zapata no debe llevar a confusión e inferir que la institución en la cual pensaba tenía las características de la biblioteca pública tal como se entiende en la actualidad: una al servicio de la comunidad. Por el contrario, por "biblioteca pública", Llano Zapata tenía en mente una destinada a la élite intelectual: la de los eruditos. Está demás decir que el proyecto del autor limeño, como tantos otros proyectos culturales en tierras peruanas, nunca prosperó.

Las razones que lo llevaron a pensar en San Marcos para el establecimiento de una biblioteca pública es una cuestión para la cual solo tengo respuestas provisionales. Aun cuando a mediados del siglo XVIII, San Marcos era pálido reflejo de lo que había sido en los siglos XVI y XVII, seguía gozando de enorme gravitación social, en particular por lo que significaba para los criollos la obtención de un grado académico: una inmejorable carta de recomendación para lograr un puesto o un ascenso en las administraciones civil y eclesiástica. ${ }^{4}$ Llano Zapata no desconocía que San Marcos, a diferencia de sus similares en el Viejo Continente, carecía de una biblioteca; de modo que poseerla le concedería mayor prestigio. También pudo pesar en su proyecto la aspiración del claustro de San Marcos por contar con una colección de libros para uso de catedráticos y alumnos, anhelo que no se había materializado por descuido del propio claustro muchos años atrás. Es conocido que, a principios del siglo XVII, el deán de la catedral de Lima, Pedro Muñiz de Medina, donó su colección de libros para el establecimiento de una biblioteca en la Universidad, pero 
que nunca llegó a realizarse, porque no hubo quien los organizara, de modo que permanecieron abandonados, expuestos al deterioro y picados por la polilla, terminaron siendo vendidos (Eguiguren, 1940-1951).

El proyecto de Llano Zapata pareció materializarse a raíz de la expulsión de la Compañía de Jesús en 1767, ya que las colecciones de libros que habían pertenecido a los colegios jesuitas fueron asignadas a seminarios y colegios en el virreinato. La importancia de los fondos bibliográficos existentes en Lima llevó a la Universidad de San Marcos a solicitar a la corona su entrega para la constitución de una biblioteca adscrita a dicha institución y bajo la custodia de sus miembros. Estos obtuvieron mediante una orden real del 25 de octubre de 1768 la cesión de los libros. Consecuentemente, las constituciones universitarias del 14 de noviembre de 1770 incluyeron las normas relativas a la organización y el funcionamiento de la proyectada biblioteca universitaria (Anónimo, 1772). Pero como la entrega de los libros se demoraba, el claustro reclamó el 25 de enero de 1778 a las autoridades locales el cumplimiento de la disposición real (Romero, 1927). En un extenso escrito señalaba que, desde tiempos muy antiguos, el principal cuidado de los monarcas había sido facilitar los medios de saber proveyendo los instrumentos necesarios para conseguirlo. Tales eran las bibliotecas públicas, porque ningún particular puede hacerse de todos los libros que necesita, aun cuando se contase con los medios económicos; ni nadie carente de recursos podía adquirir la menor parte de ellos. El claustro sostuvo que, aunque en Lima existían muchas bibliotecas particulares, eran de dominio privado y todas incompletas. Señalaban que las dos bibliotecas del antiguo colegio jesuita Máximo de San Pablo, la general y la de estudiantes, al ser principalmente de literatura eclesiástica, muy bien podían ser complementadas con los libros provenientes de otras bibliotecas de la misma congregación religiosa en cuanto "derecho civil y práctico". Estas materias, recordaban, también eran comunes porque libros sobre ellas abundaban en manos de laicos y eclesiásticos limeños. De modo que estos últimos podrían ser alentados a donarlos para la constitución de la biblioteca sanmarquina. Sentenciaban que "No necesitan menos las parroquias y monasterios de ornamentos y alhajas que el público de una biblioteca, la que no deja de ser obra piadosa porque sea decoro y ornamento de la ciudad" (Romero, 1927, pp. 31-44). Concluían señalando que el valor de las colecciones bibliográficas que pertenecieron a los jesuitas no se había de medir por el precio de sus ejemplares, porque tan grande era el número de ellos que en el caso que se vendieran, lo serían a bajo precio, lo cual terminaría atrayendo el interés de pasteleros y expendedores de especies necesitados de papel para usarlo como envoltorio. La aplicación de aquellos libros a una biblioteca pública "será la que concilie su valor" y el rey quedará "dueño de la biblioteca [...][y] también de sus intrínsecos valores” (Romero, 1927, pp. 31-44).

La necesidad de una biblioteca pública fue nuevamente expresada a finales del siglo XVIII por José Rossi y Rubí (1965), fundador y miembro de la Sociedad Amantes del País, y activo colaborador de su publicación periódica titulada Mercurio Peruano. En 1793 confesaba que, a pesar de las dificultades económicas, los miembros de la Sociedad habían decidido mantener la publicación del periódico. A la precariedad de la situación financiera se sumaba otra dificultad no menos importante: la carencia de una "biblioteca pública" (f.10). En referencia a la del Vaticano, la Ambrosiana, las reales de París y Madrid y la universitaria de Oxford, escribió, "son unos almacenes siempre abiertos, a los quales acude libremente un hombre de ingenio, sea para adquirir nuevos conocimientos, sea para rectificar los que tiene adquiridos" (Rossi y Rubí, 1965, f. 10). Como Llano Zapata, Rossi y Rubí tenía en mente el proyecto de una biblioteca al servicio del erudito. Las conversaciones domésticas, las Academias y los cafés son otros puntos de reunión "que acercan a los hombres de talento, facilitan el comercio delicioso de los descubrimientos literarios, excitan una noble emulación, publican y depuran las combinaciones científicas" sostuvo (f. 10). Pero en Lima no existían tales espacios de sociabilización, allí un escritor se encontraba aislado y, Rossi y Rubí (1965) añade, "nosotros lo hemos experimentado". Escribió que tan solo había sido gracias a los estudios realizados y la ayuda de los libros propios y prestados, que los colaboradores del Mercurio Peruano habían sido capaces de incursionar "en la dilatada y abstrusa carrera de la historia y la literatura" (f. 10). 
La falta de una biblioteca pública, sostuvo Rossi y Rubí (1965, f. 11, tenía como consecuencia la "escasez de manuscritos" de interés posibles de ser consultados por los estudiosos. Existían muchos en los archivos privados de algunos miembros de la sociedad colonial, pero estos por codicia, envidia o pereza no lo comunicaban a los académicos limeños. Sin embargo, proseguía nuestro autor, sus colegas habían adquirido a costa de su dinero algunos manuscritos. Ello representaba un sacrificio en obsequio de la patria. Una vez más, como lo hiciera Llano Zapata años antes, Rossi y Rubí reclamó como una de las tareas de la proyectada biblioteca pública la conservación de la memoria histórica escrita.

\section{LA DIMENSIÓN INSTITUCIONAL}

La materialización de una biblioteca pública en Lima se dio en el contexto de la guerra por la Independencia. Como es conocido, ante el vacío de poder creado por la abdicación de los reyes españoles a raíz de la invasión napoleónica de la península, los criollos, en diversas ciudades del continente, desconocieron la legitimidad de las autoridades coloniales, las depusieron y establecieron, en su reemplazo, juntas de gobierno. La Biblioteca Pública de Buenos Aires — antecesora directa de la Biblioteca Nacional "Mariano Moreno" - fue creada por decreto de la Primera Junta de gobierno, el 13 de septiembre de 1810. La Junta pensó que entre sus tareas estaba la de constituir modos públicos de acceso a la ilustración, visto esto como requisito para el cambio social profundo. La dirección recayó en el presbítero Luis José Chorroarín, autor de su primer Reglamento. El fondo inicial de esta Biblioteca, enlazada desde el comienzo con la lucha independentista y la refundación social, se constituyó a partir de libros procedentes de las bibliotecas del obispo Manuel de Azamor y Ramírez, del convento de La Merced y del colegio y noviciado de la Compañía de Jesús en Córdoba, así como de donaciones de particulares (Parada, 2009).

$\mathrm{Al}$ otro lado de la cordillera, en Chile, un proceso similar se dio pocos años más tarde. El 19 de agosto de 1813, la Junta de gobierno, establecida en Santiago, ordenó la recolección de libros a fin de constituir una biblioteca, ya que "el primer paso que dan los pueblos para ser sabios es proporcionarse grandes bibliotecas" (Silva Castro, 1951, p. 17. El proyecto de establecimiento de la Biblioteca Nacional no se materializó hasta la instauración del gobierno patriota luego de las acciones militares de Chacabuco y Maipú, y sobre todo durante la administración de Bernardo O 'Higgins.

Los líderes de las luchas por la Independencia fueron hombres de letras, familiarizados con la cultura escrita e impresa, y cultores de la lectura. San Martín no fue la excepción, ya que una colección de libros lo acompañó en su largo peregrinar desde Cádiz a Buenos Aires, Mendoza, Santiago de Chile y Lima. En este sentido, dos disposiciones personales constituyen muestras del valor asignado por San Martín al empleo de los libros. Cuando fue oficialmente notificado de que el Cabildo de Santiago de Chile le iba a entregar 10,000 pesos para sus gastos de viaje a las Provincias Unidas del Río de La Plata, hizo donación de ellos en una carta: "Deseoso - escribió desde Mendoza el 17 de marzo de 1817- de contribuir al lustre y esplendor de todo este reino, he designado la generosa oblación de diez mil pesos con que me ha honrado el muy ilustre cuerpo municipal [...] para que con ellos se erija una biblioteca nacional que facilite la ilustración de los ciudadanos, cuyos ingenios han sido admirados en todos tiempos" (Silva Castro, 1951, p. 31). Como finalmente el Cabildo no cumplió con la dotación de dinero, el generoso ofrecimiento del general tampoco se concretó. De igual modo, en su testamento dictado en la ciudad de Mendoza, el 23 de octubre de 1818, antes de marchar a Chile, consciente de que su vida corría peligro, expresó, por intermedio del escribano del Cabildo de esa ciudad, "Que la librería que actualmente posee y ha comprado con el fin de que se establezca y forme en esta capital una biblioteca, quede destinada a dicho fin, y se lleve a puro y decidido efecto su pensamiento" (Gutiérrez Escudero, 2007, pp. 319-337.

San Martin, acompañado de sus soldados y sus libros, llegó a la costa peruana el 9 de setiembre de 1820. Un año después, como ya se mencionó, estableció el Protectorado. En los días que siguieron a la inauguración de su gobierno, San Martín se impuso como principal tarea sentar las bases del nuevo Estado. Para tal efecto 
dictó medidas relacionadas con la demarcación política, la administración pública, el comercio de cabotaje, el ejército y la seguridad ciudadana, entre muchos otros aspectos.

Especial atención prestó el Protectorado a la instrucción, así por ejemplo, dispuso el establecimiento de escuelas de primeras letras en los conventos de frailes, y la fundación del museo y la biblioteca nacional. A pesar de su brevedad, ha escrito Juan Fonseca, el nuevo régimen se empeñó en sentar las bases de la educación pública en nuestro país, especialmente la enseñanza primaria. Entonces tenían la convicción de que la educación modelaba ciudadanos y era un medio de incorporar a los sectores populares a la naciente vida nacional. Para ello optaron por establecer un sistema educativo que no requería de una cuantiosa inversión de dinero y de personal, pero que prometía extender la "ilustración" entre los sectores populares de una manera masiva, ese fue el sistema lancasteriano (Fonseca, 2001). El fomento de la instrucción básica y la fundación de la Biblioteca Nacional pueden ser entendidas como medidas complementarias ya que ambas apuntaban a un mismo objetivo: la formación de ciudadanos.

Al decreto de fundación de la Biblioteca Nacional le siguieron otras disposiciones destinadas a consolidar la naciente institución mediante la dotación de personal, organización y rentas. Para el funcionamiento se designaron recursos económicos provenientes de la Dirección general de censos y obras pías (Puente Candamo, 1974). Los fondos bibliográficos de la Biblioteca Nacional debían estar constituidos por "todos los libros útiles que se encuentren en cualquier establecimiento público", pero no especificaba esto último. Asimismo, se ordenó que los impresores de la capital debían remitir a la Biblioteca Nacional "dos colecciones de todos los papeles públicos y demás impresos que se hayan dado a luz desde el día que se proclamó la Independencia”, y en lo sucesivo quedaban obligados a hacer lo mismo con cuánto imprimían (Puente Candamo, 1974). La medida debió parecer insuficiente para los legisladores, porque tiempo después se hizo extensiva a todos los impresores en el territorio de la república. Más aún, también se dispuso que el administrador de la aduana debía remitir a los bibliotecarios de la Biblioteca Nacional copias de las facturas de libros "que vengan para venderse en el Estado", a fin de que ellos determinen si convenía o no tomar alguna obra para la Biblioteca (Puente Candamo, 1974).

Para albergar la Biblioteca Nacional se destinó parte del edificio donde había funcionado el Colegio de la Libertad (antes del Príncipe o de caciques) y que, a raíz de la expulsión de los jesuitas, había sido trasladado al local del antiguo colegio Máximo de San Pablo. La instalación fue dispuesta e iniciada al poco tiempo de haberse aprobado el decreto legislativo; y tanto los diseños a que ella estuvo sujeta, como la ejecución de las respectivas obras, se realizaron de acuerdo con las instrucciones dadas por el ministro Bernardo de Monteagudo. El gasto inicial ascendió a 12,645 pesos, de los cuales se dieron 10,000 pesos al arquitecto Ignacio Martorel para las obras de refacción y adaptación del local; 900 pesos a José Olivares para la pintura; 1000 pesos fueron invertidos en la adquisición de "muebles, cristales y demás artículos necesarios para el estreno de la Biblioteca”; y los 745 pesos restantes fueron entregados al bibliotecario Mariano José de Arce y al conservador José Valerio Gasols, para los gastos menudos que requería la instalación (Tauro, 1951, p. 22).

El fondo bibliográfico inicial de la Biblioteca Nacional estuvo constituido por los libros donados por el general San Martin, así como por Martín de Osambela, Pedro Mariano Goyeneche y Miguel de la Fuente, entre otros vecinos de Lima. Asimismo, la colección de San Marcos y muchos otros libros procedentes de “Monsarrat" pasaron a la nueva institución (Anónimo, 1971; Tschudi, 2003). También San Martín instruyó a sus ministros plenipotenciarios acreditados en Francia e Inglaterra -Juan García del Río y Diego Paroissien -, para que adquiriesen libros "modernos" (Tauro, 1951).

Mientras las obras de instalación de la Biblioteca Nacional avanzaban, el régimen del Protectorado de San Martín entraba en la última etapa de su existencia. A fines de julio de 1822, San Martín se reunió con Simón Bolívar en Guayaquil. Aunque, los términos de las conversaciones siguen siendo un misterio, San Martín al parecer habría pedido ayuda militar a El Libertador con el fin de poner fin a la guerra contra el ejército del rey. Lo más probable es que no hubiera un acuerdo entre ambos militares, ya que entre ellos existía notables diferencias políticas: San Martín era partidario de implantar un régimen monárquico constitucional en el Perú, mientras que Bolívar veía con buenos ojos la idea de un régimen republicano. 
San Martín regresó a Lima, para encontrar que su autoridad se había debilitado fuertemente como consecuencia de la cada vez más creciente oposición política a sus planes de gobierno para el naciente Estado peruano y, en particular, el desencanto de la élite debido a las exacciones cometidas para sufragar la guerra. Peor aún, su ministro y principal colaborador, Monteagudo, había sido depuesto, por lo que, ante tal situación, decidió renunciar al Protectorado (Hernández García, 2019). San Martín, deseoso de acabar algunas de las tareas aú pendientes, procedió el 17 de setiembre a inaugurar la Biblioteca Nacional. Un riguroso protocolo rigió 1 ceremonia que presidió y contó con la asistencia de connotadas figuras adel régimen. El 20 del mismo mes entregó el mando al Congreso Constituyente, que él mismo había convocạdo pocos días antes y ese día partió rumbo a Chile.

\section{LA DIMENSIÓN SIMBÓLICA}

Además de tener una dimensión institucional, la fundación de la Biblioteca Nacional tiene una dimensión simbólica plasmada en los discursos: constituía una ruptura con el pasado. Antes de su establecimiento, en Lima, como en el resto de ciudades de la América colonial, las bibliotecas eran privadas, bien en manos de instituciones religiosas o de particulares, como ya se ha dicho. El decreto de San Martín establecía una "biblioteca pública" con carácter de "nacional". No se trataba de una biblioteca destinada a un público erudito, tal como la habían concebido Llano Zapata y Rossi y Rubí, sino al conjunto de la sociedad. Así lo declara el decreto fundacional al señalar que es "para el uso de todas las personas que gusten concurrir a ella”. Por añadidura, la asociación entre nación y sociedad es manifiesta en el lenguaje del régimen sanmartiniano. Así, en el "Preámbulo" del Estatuto Provisorio de 1821, el general rioplatense expresa: "Me he encargado de toda la autoridad, para responder de ella a la nación entera" (Fernández Sebastián, 2009, p. 946). Asimismo, la voluntad de ruptura con el régimen colonial se expresó en el léxico.

Antes de la llegada de la expedición libertadora, las poblaciones de la costa, entre ellas Lima, habían sido invadidas por abundante propaganda impresa patriota. En su afán por justificar la causa, los partidarios de la Independencia emplearon un lenguaje condenatorio del régimen colonial. Aunque la crítica a este último era muy antigua, se la puede retrotraer al siglo XVI, el discurso patriota de inicios de la década de 1820 es heredero del empleado por el régimen liberal español establecido por las Cortes de Cádiz años atrás. Entonces, las instituciones y los principios de la monarquía española fueron puestos en entredicho públicamente al ser discutidos en la prensa periódica y en una prolífica bibliografía.

La idea de un régimen despótico que mantuvo a los americanos en la ignorancia, alejados del desarrollo del conocimiento es un tópico recurrente de la retórica patriota. El decreto de fundación de la biblioteca es buena muestra de ello. Allí se dice, que convencido el gobierno español de que la ignorancia era la columna más firme del despotismo, "puso las trabas más fuertes a la ilustración del poblador americano, manteniendo su pensamiento encadenado para impedir que adquiriese el conocimiento de su dignidad" (Puente Candamo, 1974, p. 294). Los gobiernos libres — prosigue el mismo decreto- que se han constituido sobre las ruinas de la tiranía, debian adoptar otro sistema enteramente distinto, dejando a los hombres el camino hacia la perfección. Era tarea de toda "administración ilustrada" facilitar a los pobladores los medios para acrecentar sus "luces" y fomentar su «civilización» por medio de establecimientos útiles. Gracias a estos últimos:

las almas reciben [...] nuevo temple, toma vuelo el ingenio, nacen las ciencias, disípanse las preocupaciones [...], propáganse los principios conservadores de los derechos públicos y privados, triunfan las leyes y la tolerancia y empuña el cetro la filosofía, principio de toda libertad, consoladora de todos los males, y origen de todas las acciones nobles (Puente Candamo, 1974, p. 295).

La idea regeneradora de la cultura volvió a ser retomada en el decreto de funcionamiento de la Biblioteca Nacional promulgado el 8 de febrero de 1822. Allí se dice que su establecimiento es uno de los medios más eficaces para "poner en circulación los valores intelectuales, y hacer que los hombres de todas las edades 
se comuniquen recíprocamente los secretos que han escudriñado en el fondo de la naturaleza" (Puente Candamo, 1974, p. 295). En los considerandos del primer Reglamento de la Biblioteca Nacional, fechado el 31 de agosto de 1822, se reitera el tópico negativo acerca de la realidad cultural del virreinato. Se dice que en "un país que, habiendo sido bajo el sistema español el centro del despotismo y de la arbitrariedad, se han escaseado por una funesta política todos los recursos de la ilustración, prohibiendo la lectura de libros selectos y el estudio de las ciencias relativas a los derechos del hombre" (Puente Candamo, 1974, p. 297). Nada más alejado de la realidad, porque como ya se ha dicho antes, desde una época temprana de la colonización española circularon libros entre los miembros de la sociedad colonial.

En el discurso de la ceremonia de inauguración de la Biblioteca Nacional, su director, Francisco Valdivieso, quien era también ministro de Estado y de Relaciones Exteriores, sostuvo que se complacía de que en Lima existiese una "fuente de abundante instrucción pública, de donde deben salir los verdaderos principios de prosperidad de estos privilegiados países y las sólidas bases de engrandecimiento a que los llama su destino" (Universidad Nacional de la Plata, 1950, p. 665). Ese día, prosiguió Valdivieso, "es mui amargo para nuestros enemigos, mui dulce para la Patria". Y añadió que con la inauguración "se anuncia el triunfo de las luces que harán siempre invencible las armas de la América, y la libertad, fruto precioso de su constancia y sacrificios" (p. 665). Por su parte, San Martín se refirió a que la nueva institución estaba destinada a la "ilustración universal, más poderosa que nuestros ejércitos, para sostener la Independencia". A lo que agregó en tono esperanzador: "Los cuerpos literarios deben fomentar aquella, concurriendo sus individuos a la lectura de los libros, para estimular a lo general del pueblo a las delicias del estudio". Y concluyó con las siguientes palabras: "Yo espero que así sucederá; y que este establecimiento, fruto de los desvelos del gobierno, será frecuentado por los amantes de las letras y de su patria” (p. 665). De modo que el discurso patriota además de condenar el pasado, tenía una finalidad esencial: enfatizar el inicio de una nueva época, en la que, para usar su propio léxico, las "luces" del conocimiento irradiasen al conjunto de la sociedad.

Los primeros años de vida de la Biblioteca Nacional fueron, por decirlo de alguna manera, azarosos. La Biblioteca fue saqueada por el ejército realista durante su breve ocupación de la capital en julio de 1823 (Anna, 1979; Núñez, 1971). En cualquier caso, el más temible enemigo de la institución —ayer, hoy y siempre- es aquel usuario que cree tener derecho de posesión sobre los bienes públicos. En 1825, el gobierno denunció que "faltan muchas y preciosas obras de la Biblioteca Nacional". En consecuencia, y siendo necesario tomar medidas necesarias para lograr su restitución, ordenó que todos los individuos que tenían libros pertenecientes a la biblioteca "vendidos por españoles o adquiridos de cualquier modo que no sea por enajenación del gobierno”, los debían entregar al ministerio de gobierno, sin excusa alguna, y en caso de no hacerlo, serían multados con 500 pesos (Santos de Quirós y Nieto, 1831-1854, II, p. 101). La historia de pérdidas y sustracciones desafortunadamente no es nueva.

\section{CONCLUSIONES}

La tarea de reconstruir la historia de la Biblioteca Nacional constituye un fascinante ejercicio académico, ya que nos permite recuperar las voces del pasado y entender en perspectiva histórica los proyectos, los anhelos y los fracasos de los gestores de las políticas culturales en nuestro país, así como también evaluar cuánto hemos avanzado en lo que alguna vez Jorge Basadre (1958) llamó "la promesa de la vida peruana". Los fundadores del Estado peruano estuvieron animados de ideales, bienestar y prosperidad para la sociedad. La creación de la Biblioteca Nacional se inscribe dentro de esa propuesta. Se trató, como se ha visto, de un hito en el proceso de constitución del nuevo Estado, ya que fue la primera institución de su tipo, que pretendía estar abierta al público sin distinciones. Al mismo tiempo, significó una ruptura con el régimen colonial de acuerdo con los ideólogos de la Independencia, quienes vieron en ella el inicio de una época de difusión de la cultura, la cual debía contribuir no solo al mejor conocimiento, sino además a la formación de ciudadanos. 


\section{Agradecimientos}

Agradezco a Carlos Aguirre y a los evaluadores anónimos por su lectura y comentarios a la versión preliminar de este texto.

\section{REFERENCIAS}

Anna, T. (1979.The fall of Royal government in Perú. Lincoln: Univeristy of Nebraska Press.

Anónimo. (1772. Colección de las aplicaciones que se van haciendo de los bienes, casas, y colegios que fueron de los regulares de la Compañia de Jesús expatriados de estos reales dominios: siguiendo en todo lo adaptable las reglas que prescribe la Real Cédula dada en Madrid a 9 de julio de 1769. Lima: Oficina de la calle San Jacinto.

Anónimo. (1971). Documentos referentes a la Biblioteca Nacional: 1822-25, 1829. Boletín de la Biblioteca Nacional, 59-60, 5-11.

Basadre, J. (1958). La promesa de la vida peruana y otros ensayos. Lima: Juan Mejía Baca.

Cama, J. y Cama, M. (2010). La Ilustración en Lima. Vida y obra del doctor Cosme Bueno y Alegre (1711-1798). Lima: Centro de Producción Editorial e Imprenta de la Universidad Nacional Mayor de San Marcos.

Chartier, R. (1993). Libros, lecturas y lectores en la edad moderna. Madrid: Alianza Editorial.

Crawford, A. (Ed.) (2015). The meaning of the library. A cultural history. Princeton: Princeton University Press.

Darnton, R. (2015). From printing shop to bookshelves: how books began the journey to enlightenment libraries. En A. Crawford (Ed.). The meaning of the library. A cultural history (pp. 91-102). Princeton: Princeton University Press.

Eguiguren, L. A. (1940-1951). Diccionario histórico cronológico de la universidad real y pontificia de San Marcos y sus colegios. Crónica e investigación. Lima: Torres Aguirre, 3 vols.

Fernández Sebastián, J. (Ed.). (2009). Nación - Perú. En Diccionario politico y social del mundo iberoamericano. La era de las revoluciones, 1750-1850. Madrid: Fundación Carolina, Sociedad Estatal de Conmemoraciones Culturales y Centro de Estudios Políticos y Constitucionales.

Fonseca, J. (2001). Sin educación no hay sociedad: Las escuelas lancasterianas y la educación primaria en los inicios de la república (1822-1826). En S. O'Phelan (Comp.). La Independencia del Perú. De los borbones a Bolivar (pp. 265-287). Lima: Instituto Riva-Agüero.

Guibovich Pérez, P. (2003). Los espacios de los libros en el Perú colonial. Lexis, 27/1-2, 179-190.

Guibovich Pérez, P. (2014). Tradición y modernidad. La biblioteca del obispo Pedro José Chávez de la Rosa. Lima: Instituto Riva-Agüero.

Guibovich Pérez, P. (2019). Society and education: the University of San Marcos in the 16th century. En E. Engel (Ed.). A companion to early modern Lima (pp. 216-234). Leiden/Boston: Brill.

Gutiérrez Escudero, A. (2007). José de San Martín. Rasgos de su personalidad. Araucaria, 18, 319-337. Recuperado de https://www.redalyc.org/pdf/282/28291818.pdf

Hernández García, E. (2019). José de la Riva-Agüero (1783-1858), primer presidente del Perú. Lima: Fondo Editorial del Congreso del Perú e Instituto Riva-Agüero.

Llano Zapata, J. E. (2005). Memorias histórico, físicas, críticas, apologéticas de la América meridional. Lima: Universidad Nacional de Mayor de San Marcos, Pontificia Universidad Católica del Perú e Instituto Francés de Estudios Andinos.

López Vidriero, M. (1994). La imprenta en el siglo XVIII. En Historia ilustrada del libro español. II. De los incunables al siglo XVIII (pp. 201-269). Madrid: Fundación Germán Sánchez Ruipérez.

Novoa, M. (2007). El saber legal a inicios de la Independencia: la cultura jurídica de Manuel de Arredondo (c.1738-1822), regente de Buenos Aires y Lima. En C. McEvoy y A. Stuven (Eds.). La República Peregrina. Hombres de armas y letras en América del sur, 1800-1884 (pp. 39-75). Lima: Instituto de Estudios Peruanos e Instituto Francés de Estudios Andinos. 
Núñez, E. (1971). Mariano José de Arce, primer bibliotecario. Fénix, 21, 46-57.

Parada, A. E. (2009). Los orígenes de la Biblioteca Pública de Buenos Aires. Antecedentes, prácticas, gestión y pensamiento bibliotecario durante la revolución de mayo (1810-1826). Buenos Aires: Instituto de Investigaciones Bibliotecológicas de la Universidad de Buenos Aires.

Puente Candamo, J. A. de la (Comp.). (1974). Colección documental de la Independencia del Perú. T. XIII. Obra de gobierno y epistolario de San Martín. Volumen I. Lima: Comisión del Sesquicentenario de la Independencia del Perú.

Romero, C. (1927). La biblioteca de la Universidad de San Marcos y el bibliotecario fray Diego Cisneros. Boletín Bibliográfico, 3(2), 31-44.

Rossi y Rubí, J. (1965). Concluye la Introducción al tomo VII. Mercurio Peruano del día 6 de enero de 1793. Edición facsimilar. Lima: Biblioteca Nacional del Perú.

Santos de Quirós, M. y Nieto, J. C. (1831-1854). Colección de leyes, decretos y órdenes publicadas en el Perú desde su independencia. Lima: Imprenta de Juan Masías.

Saravia, R. (2020). Bernardo de Monteagudo, primer director de la Biblioteca Nacional del Perú. Fénix, 48, 9-28. Recuperado de http://revistafenix.bnp.gob.pe/index.php/fenix/article/view/570

Shafer, R.J. (1958).The economic societies in the Spanish world (1763-1821). New York: Siracuse University Press.

Silva Castro, R. (1951). Los primeros años de la Biblioteca Nacional (1813-1824). Santiago de Chile: Sociedad de Bibliófilos Chilenos.

Solís, J. (1993). La idea de la biblioteca pública en tiempos del Mercurio Peruano. Boletín del Instituto Riva-Agüero, 20, 56-64.

Tauro, A. (1951). La fundación de la Biblioteca Nacional. Anuario bibliográfico peruano de 1948, 9-41.

Tschudi, J. J. (2003). El Perú. Esbozos de viajes realizados entre 1838 y 1842. Lima: Pontificia Universidad Católica del Perú.

Universidad Nacional de la Plata. (1950). Gaceta del gobierno de Lima independiente. La Plata: Universidad Nacional de La Plata.

Walker, Ch. (2005). Prólogo. En J.E. Llano Zapata. Memorias histórico, físicas, críticas, apologéticas de la América meridional (pp. 21-36). Lima: Universidad Nacional de Mayor de San Marcos, Pontificia Universidad Católica del Perú e Instituto Francés de Estudios Andinos.

\section{Notas}

1 Aunque un poco antigua e imprecisa, la síntesis de Macera (1966) sobre bibliotecas coloniales sigue siendo útil. Acerca de bibliotecas particulares de fines del siglo XVIII e inicios del XIX, véase Novoa (2007), Cama y Cama (2010) y Guibovich Pérez (2014).

2 Durante la ocupación militar de Lima por el ejército chileno entre 1881 y 1883, la Biblioteca Nacional fue entrada a saco y muchos de sus libros y manuscritos trasladados como botín de guerra a Chile. Años después, en 1943, un incendio destruyó la biblioteca. Estos dos eventos explican la carencia de un archivo institucional.

3 Sobre los cuidados dados a los libros, véase Guibovich Pérez (2003).

4 Para una historia de la trayectoria de San Marcos en el siglo XVI, véase Guibovich Pérez (2019). 